\begin{tabular}{|c|l|}
\hline Title & Macroscopic motion of supramolecular assemblies actuated by photoisomerization of azobenzene derivatives \\
\hline Author(s) & $\begin{array}{l}\text { Kagey ama, Y oshiyuki; T anigake, Naruho; Kurokome, Y uta; I waki, Sachiko; Takeda, Sadamu; Suzuki, Kentaro; } \\
\text { Sugawara, Tadashi }\end{array}$ \\
\hline Citation & $\begin{array}{l}\text { Chemical communications, 49(82), 9386-9388 } \\
\text { https://doi.org/10.1039/c3cc43488e }\end{array}$ \\
\hline Issue Date & 2013-06-08 \\
\hline Doc URL & http://hdl.handle.net/2115/57294 \\
\hline Type & article (author version) \\
\hline File Information & kageyama2013-12-18.pdf \\
\hline
\end{tabular}

Instructions for use 


\title{
Macroscopic Motion of Supramolecular Assemblies Actuated by Photoisomerization of Azobenzene Derivatives
}

\author{
Yoshiyuki Kageyama, ${ }^{a}$ Naruho Tanigake, ${ }^{a}$ Yuta Kurokome, ${ }^{a}$ Sachiko Iwaki, ${ }^{a}$ Sadamu Takeda, $*^{a}$ \\ Kentaro Suzuki, ${ }^{b}$ and Tadashi Sugawara ${ }^{* b}$
}

\author{
5 Received (in $X X X, X X X)$ Xth $X X X X X X X X X 20 X X$, Accepted Xth $X X X X X X X X X 20 X X$ \\ DOI: $10.1039 / b 000000 x$
}

Submillimetre size self-assemblies composed of oleate and azobenzene derivatives show forceful motions such as screwtype coiling-recoiling motion by photoirradiation.

10 In the past half century, many molecular-level machines which change their molecular structure upon exposure to stimuli have been reported. ${ }^{1}$ Nowadays, studies on macroscopic thermal motion of soft matters received attentions from the viewpoint of biomimetics. $^{2,3}$ Next key step for soft matters is to create a 15 material whose macroscopic motion is controlled by external stimuli. $^{4}$ One of the strategies is to create soft materials consisting of subunits sensitive to external inputs, for instance, the photoinduced bending of polymer films consisted of azobenzene derivatives. ${ }^{5,6}$ Another strategy is to create soft 20 materials in which macroscopic motions of molecular assemblies are actuated by small amount of molecular machines. ${ }^{7}$ To date, there are few reports describing macroscopic mechanical behaviour based on the latter strategy. ${ }^{8}$ Here, we report reversible and spatially controlled macroscopic motion of oleate 25 assembles which have submillimetre length.

Mixtures of oleic acid (1H) and oleate (1) (Fig. 1) are capable of self-assembly in aqueous solution. The morphology of the mixture depends on $\mathrm{pH}$ and ionic strength $(I) .{ }^{9-11} \mathrm{In} I=0.1 \mathrm{pH} 8^{-}$ 10 aqueous media, oleates form vesicles (Fig. 2a). Between $\mathrm{pH} 7$ 30 and 8, they form several complex aggregates called "blocks" that exhibit an inverted hexagonal phase ${ }^{12}$ (Fig. 2b). In the transition region, helical and straight multilayer assemblies are coexisted with vesicles and blocks. ${ }^{11}$ It is considered that the shape of assembly depends on the ratio of $\mathbf{1 H}$ and $\mathbf{1}^{-}$in each assembly. 35 According to the microscopic observations of shape and dynamics described in Ref. 11, the helical assemblies are sufficiently flexible to change their macroscopic helical pitch and curvature, but are also elastic because of the highly-ordered stacking structure of oleate molecules in the assembly. As 40 demonstrated in Fig. 3, the helical assemblies exhibit a highly elastic response to mechanical stress. We assumed that this softness and elasticity of the self-assembly is suitable to the spatially moving material in which motion of molecular machine should be propagated.

45 Azobenzene derivatives 2-5 (Fig. 1) display reversible photochromic behaviour under exposure to $366 \mathrm{~nm}$ UV light ( $E-Z$ isomerization) and $435 \mathrm{~nm}$ blue light $\left(Z-E\right.$ isomerization) ${ }^{13}$ Preparation of the assemblies generally involved hydration of a mixture of $4.5 \mathrm{mg}$ sodium oleate (1Na) and $0.45 \mathrm{mg}(10 \mathrm{wt} \%)$ of

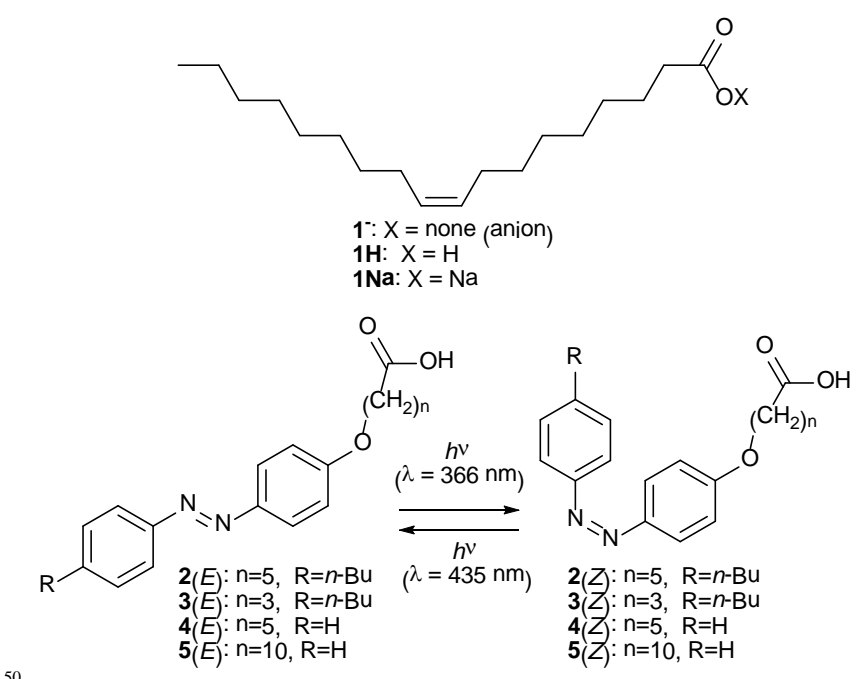

Fig. 1. Compounds and their photoisomerization.
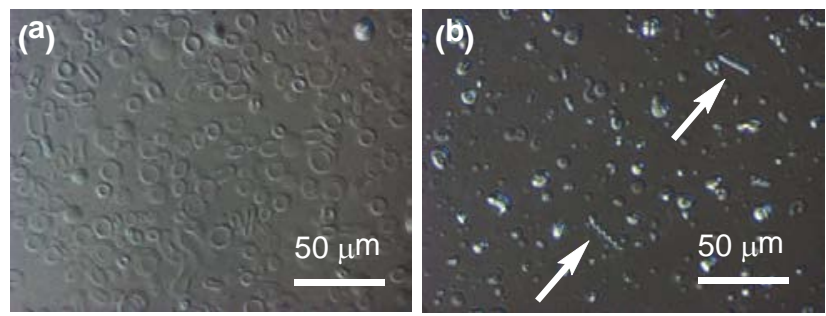

Fig. 2. Differential interference contrast (DIC) microscopic images of $\mathrm{pH}$ dependent oleate assemblies: (a) giant vesicles ( $\mathrm{pH} 8$ ), (b) blocks and 55 helical structures (indicated by arrows) in $\mathrm{pH} 7.8$ dispersion ( $0.1 \mathrm{M}$ aq. $\mathrm{NaCl})$.

2-5 by addition of $70 \mathrm{mM} \mathrm{KH} \mathrm{PO}_{4}-\mathrm{K}_{2} \mathrm{HPO}_{4}$ buffer solution $(1.0 \mathrm{~mL})$ and sonication for $20 \mathrm{~min}$. A $125 \mu \mathrm{L}$ aliquot of each suspension containing one of $\mathbf{2}-\mathbf{5}$ was placed in a sealed 60 microscope slide and incubated for 1 day at $25^{\circ} \mathrm{C}$. All azobenzene derivatives dissolved into oleate assemblies. The $\mathrm{pH}$ range required for formation of helical assemblies was slightly lowered by the addition of $\mathbf{2}-\mathbf{5}^{14}$ Helical assemblies were formed when $\mathrm{pH} 7.3$ buffer solution was used as the dispersing 65 medium (the $\mathrm{pH}$ of the dispersion was approximately 7.5); $\mathrm{pH}$ values of buffer solution higher than 7.3 resulted in vesicle formation, and $\mathrm{pH}$ values lower than 7.3 led to the formation of 
blocks.

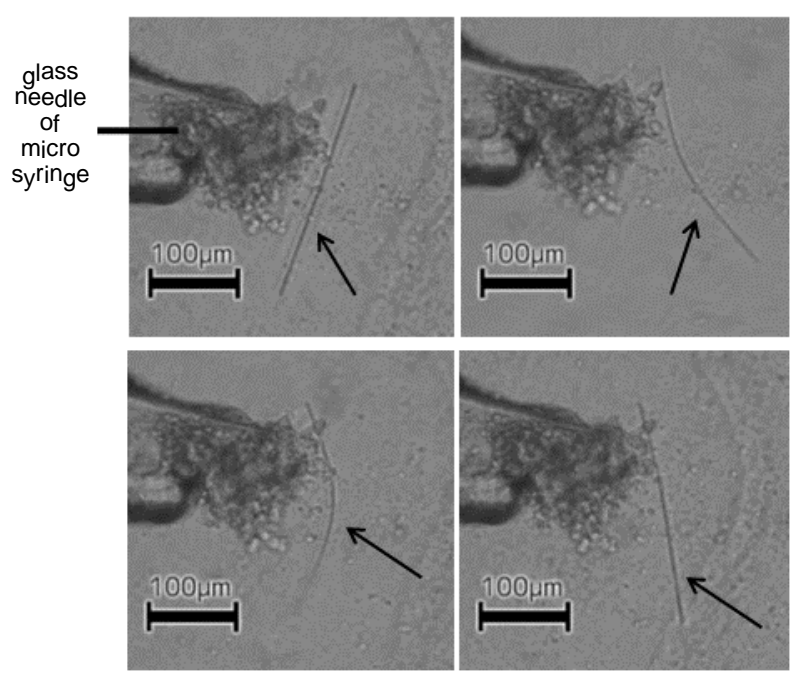

Fig. 3. Elasticity of helices responding to mechanical contact with a glass needle observed by a phase contrast microscope
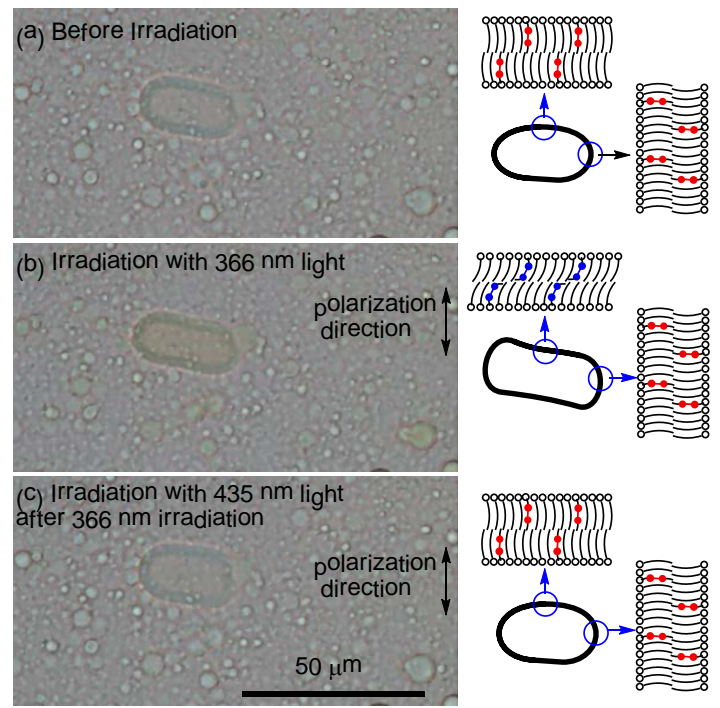

Fig. 4. Reversible deformation of giant vesicles induced by photoisomerization of azobenzene derivatives (pH 7.8) observed by DIC microscopy (movie 1 in ESI): (left) micrographs showing ellipsoidal deformation of several vesicles; (right) schematic illustration of anisotropic deformation of vesicles induced by polarized light (red indicates trans-form of azobenzene, and blue indicates cis-form of azobenzene.). The photoisomerization of azobenzenes in the lateral sides of the vesicles is less efficient than that of azobenzenes located on the upper and lower sides of the vesicles. ${ }^{16}$

15 First we examined the effect of UV-light irradiation of the 2containing vesicular dispersion at $\mathrm{pH}$ 7.8. Irradiation with 366 $\mathrm{nm}$ light through an objective lens from an incident light component of a fluorescence microscope induced a slight increase in the size of vesicles. The vesicle restored to the 20 original size by irradiation with $435 \mathrm{~nm}$ light. This reversible change in size is associated with the photochromic behaviour of the azobenzene derivatives. A unique change in vesicular shape was observed more distinctively when using polarized light. They deformed in an ellipsoidal manner by irradiation with light 25 of 366 nm wavelength polarized longitudinally in Fig. 4 and in movie 1 contained in the Electronic Supplementary Information (ESI). Because the optical transition moment is parallel to the
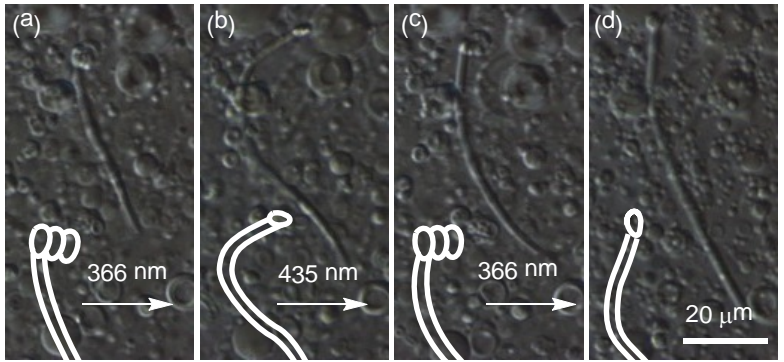

Fig. 5. DIC micrographs of reversible motion of multilayer assembly (pH 30 7.5) with illustrations (movie 2 in ESI). The helical structure (top of a) was converted to a straight assembly following $366 \mathrm{~nm}$ irradiation (b), recoiled to a helical form following $435 \mathrm{~nm}$ irradiation (c), and straightened following $366 \mathrm{~nm}$ irradiation (d).

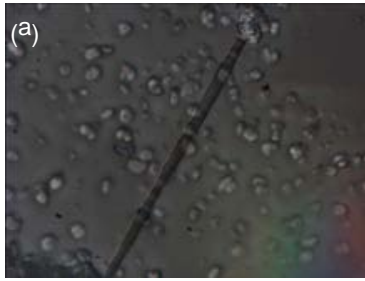

(c)
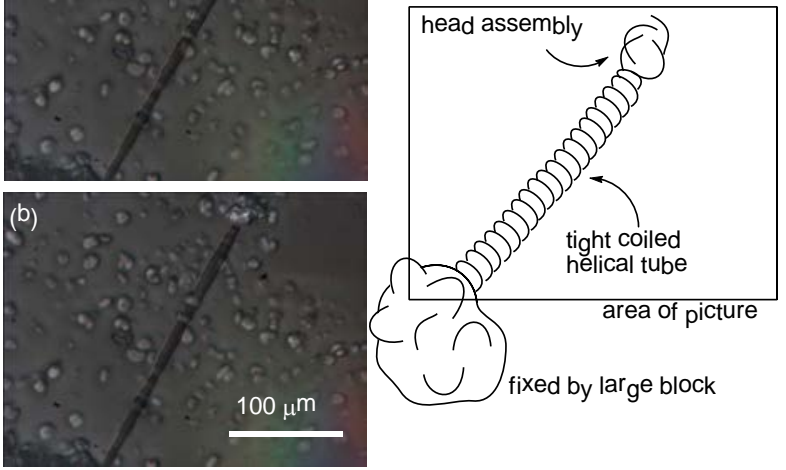

35 Fig. 6. Rotation motion of tightly coiled helical assembly composed of oleate and $10 \mathrm{wt} \%$ of 3 by $366 \mathrm{~nm}$ irradiation (pH 7.4), observed by DIC microscopy (a) and (b), and illustration for description (c): (b) is the picture taken just after (a) under irradiation of $366 \mathrm{~nm}$ light. The change of the angle of the head assembly indicates the rotation of the

40 helicalassembly. The reverse rotation under irradiation of $435 \mathrm{~nm}$ was also observed, see movie 3 in ESI. ${ }^{17}$

long axis of the azobenzene chromophore, ${ }^{15}$ this photoinduced deformation indicates that the azobenzene is oriented vertically against the membrane surface, and that azobenzene molecules on 45 the upper and lower vesicular membrane surfaces absorbed light more efficiently than those on lateral surfaces (Fig. 4). ${ }^{16}$ Next, we examined the effect of light irradiation on the block-type aggregates containing 2 . In contrast to vesicles, the response of block-type aggregates was less intense, suggesting that the 50 inverted hexagonal structure is difficult for propagating the structural change, compared with the lamellar structure of vesicles. Similar behaviours were observed in the assemblies containing 3-5.

The highly ordered helical or straight multilayer assemblies 55 containing the azobenzene derivative (2-5) exhibited forceful motion. Irradiation of a bold helical assemble with $366 \mathrm{~nm}$ light resulted in straightening of the helical assemble, and irradiation of the straightened assemble with $435 \mathrm{~nm}$ light re-coiled it to the helical structure (Fig. 5 and movie 2 in ESI). This uncoiling 60 (straightening) and coiling motions were seen as rotationrerotation motion in tightly coiled helical assemblies (Fig. 6 and 
movie 3 in ESI). ${ }^{17}$ As far as we know, this type of macroscopic propelling motion has not been reported in artificial supramolecular chemistry.

We also observed the photoinduced macroscopic motion of 5 oleate assemblies containing smaller amount of azobenzene derivatives (1 wt\% of 2). Unfortunately, rapidly and efficiently responded dynamics were not observed. However, interestingly, after continuous irradiation with $366 \mathrm{~nm}$ light for over $20 \mathrm{~min}$, macroscopic motions suddenly occured. ${ }^{18}$ Because the change 10 was reversed by $435 \mathrm{~nm}$ light, it is considered that the change is not the result of thermal effect of long-term irradiation but the photoisomerization of 2 . The extended induction period after irradiation suggests that the morphological changes occur only after substantial amounts of $\mathbf{2}$ have been isomerized. In other 15 words, some kinds of cooperation work among photoisomerized azobenzenes dispersed in oleate assembly and induce the macroscopic motion. ${ }^{19}$

The forceful motion exhibited by the helical assemblies and the presence of an induction period strongly suggest there are 20 cooperatively propagating mechanisms such as domino effects occurring inside or at the surface of the supramolecular assemblies. We assume that the change of effective volume of azobenzene units by photoisomerization causes the propagation of expansion or contraction of inter-molecular geometry among 25 oleate molecules. This change of effective molecular volume by light irradiation may occur and take an important role to orientation of molecules in the soft molecular assemblies, in which anisotropic molecular rotation and water diffusion takes place. We are trying to get an evidence to prove this hypothesis 30 experimentally. The result presented here is noteworthy, since it is a demonstration that the microscopic motion of diluted small molecules can create macroscopically observable effects in supramolecular assemblies. Observed macroscopic motional mode depended on the morphology of molecular assemblies, such 35 as vesicles, helical assemblies, and blocks. This result indicates the creation of highly organized motions is possible by designing the morphology of molecular assemblies. From these viewpoints, this work is of great importance in reference to the construction of supramolecular machinery.

40 This work was funded by the Sasakawa Scientific Research Grant from The Japan Science Society, JSPS KAKENHI (No. 23750142 and 25620002), and Global COE (Catalysis as the Basis for Innovation in Material Science) from MEXT Japan. K.S. and T.S. acknowledge the support from KAKENHI on ${ }_{45}$ Priority Area "Soft Matter Physics" (MEXT).

\section{Notes and references}

${ }^{a}$ Department of Chemistry, Faculty of Science, Hokkaido University, Sapporo 060-0810, Japan. E-mail: stakeda@sci.hokudai.ac.jp,

50 y.kageyama@mail.sci.hokudai.ac.jp; Fax: +81-11-706-4841; Tel: +8111-706-3505

${ }^{b}$ Department of Chemistry, Faculty of Science, Kanagawa University, Hiratsuka, Kanagawa 259-1293, Japan. Tel: +81 463-59-4111; E-mail: sugawara-t@kanagawa-u.ac.jp

$55 \dagger$ Electronic Supplementary Information (ESI) available: materials, Fig. S1 and S2, and movie1-3. See DOI: 10.1039/b000000x/

1 E. R. Kay, D. A. Leigh and F. Zerbetto, Angew. Chem. Int. Ed., 2007, 46, 72-191; J. Michl and E. C. H. Sykes, ACS Nano, 2009, 3, 10421048 .
602 R. V. Craster and O. K. Matar, Rev. Mod. Phys., 2009, 81, 11311198; K. Suzuki, T. Toyota, K. Takakura and T. Sugawara, Chem. Lett., 2009, 38, 1010-1015; G. Zhao and M. Pumera, Chem. Asian J., 2012, 7, 1994-2002.

3 M. M. Hanczyc, T. Toyota, T. Ikegami, N. Packard and T. Sugawara, 65 J. Am. Chem. Soc., 2007, 129, 9386-9391; K. Suzuki, R. J. Akthar, H. Mahara, H. Hashimoto, T. Iwatsubo, S. Nishimura and T.Yamaguchi, Chem. Lett., 2009, 38, 20-21.

4 K. Ariga, T. Mori and J. P. Hill, Chem. Sci.,. 2011, 2, 195-203.

5 D. Bléger, Z. Yu and S. Hecht, Chem. Commun., 2011, 47, 1226012266.

6 Y. Yu, M. Nakano and T. Ikeda, Nature, 2003, 425, 145; N. Hosono, T. Kajitani, T. Fukushima, K. Ito, S. Sasaki, M. Tanaka and T. Aida, Science, 2010, 330, 808-811; K. M. Lee, H. Koerner, D. H. Wang, L.-S. Tan, T. J. White and R. A. Vaia, Macromolecules, 2012, 45, $757527-7534$; N. Hosono, M. Yoshikawa, H. Furukawa, K. Totani, K. Yamada, T. Watanabe and K. Horie, Macromolecules, 2013, 46, 1017-1026.

7 J. M. Spruell and C. J. Hawker, Chem. Sci., 2011, 2, 18-26; As a recent example, J. Ryu, M. D’Amato, X. Cui, K. N. Long, H. J. Qi and M. L. Dunn, Appl. Phys. Lett., 2012, 100, 161908.

8 Macroscopic motions of liquid droplets caused by structural change of molecules were reported in: K. Ichimura, S.-K. Oh and M. Nakagawa, Science, 2000, 288, 1624-1626; J. Berná, D. A. Leigh, M. Lubomska, S. M. Mendoza, E. M. Perez, P. Rudolf, G. Teobaldi and 85 F. Zerbetto, Nature Mater., 2005, 4, 704-710; P. Wan, Y. Jiang, Y. Wang, Z. Wang and X. Zhang, Chem. Commun., 2008, 44, 57105712.

9 J. M. Gebicki, and M. Hicks, Nature, 1973, 243, 232-234; K. Edwards, M. Silvander and G. Karlsson, Langmuir, 1995, 11, 2429$90 \quad 2434$; K. Morigaki and P. Walde, Curr Opin Colloid Interface Sci., 2007, 12, 75-80.

10 B. Dejanović, V. Noethig-Laslo, M. Šentjurc. P. Walde, Chem. Pys. Lipids, 2011, 164, 83-88; H. Fukuda, A. Goto, H. Yoshioka, R. Goto, K. Morigaki and P. Walde, Langmuir, 2001, 17, 4223-4231.

9511 M. Ishimaru, T. Toyota, K. Takakura, T. Sugawara and Y. Sugawara, Chem. Lett., 2005, 34, 46-47.

12 By our preliminary microbeam small angle X-ray scattering utilized KEK PF-BL4A, hexagonal patterns and layer patterns were detected from block-type assemblies. The $d$-spacings values were depended $100 \quad$ on $\mathrm{pH}$ and were ranged $5.3(\mathrm{pH} 7.7)-5.9(\mathrm{pH} \mathrm{8.0)} \mathrm{nm}$.

13 Synthesis of $\mathbf{2}-\mathbf{5}$ and changes occurring in the UV-Vis spectra during photoisomerization of $\mathbf{2}$ are shown in ESI.

14 Generally, the morphology of self-assembly in aqueous media depends on the packing parameters of amphiphiles (J. N. Israelachvili, 105 D. J. Mitchell, B. W. Ninham, J. Chem. Soc., Faraday Trans. 2, 1976, 72, 1525-1568). We assumed that one of the significant reasons for the shift of $\mathrm{pH}$ regions for helical assemblies formation is a change of averaged packing parameter by addition of azobenzene derivatives

15 J.-M. Pedrosa, M. T. M. Romero, L. Camacho and D. Möbius, J. 110 Phys. Chem. B, 2002, 106, 2583-2591.

16 The magnitude of the morphological changes was smaller when nonpolarized light was irradiated, suggesting that the slower volume change of water in the interior restricts vesicular motion. The same rationale may be used to explain the inequality of response between

115 the sides of the vesicles perpendicular and parallel to the incident light.

17 The experimental method was described in ESI.

18 We observed vesicular membrane formed from multilamellar assembly by $366 \mathrm{~nm}$ light (Figure S2). We could not define the 120 induction time because it was dependent on the nature of the assemblies.

19 In a manner similar to the assemblies containing $1 \mathrm{wt} \%$ azobenzene derivative, there were short induction times prior to observation of morphological transformations in assemblies containing $10 \mathrm{wt} \%$ of 2 when exposed to weak light. 


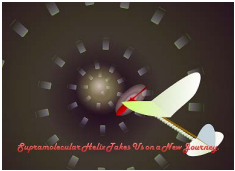

